\title{
Original
}

\section{Clinical impact of women with gestational diabetes mellitus by the new consensus criteria: two year experience in a single institution in Japan}

\author{
Satoru Ikenoue $^{1)}$, Kei Miyakoshi ${ }^{1)}$, Yoshifumi Saisho ${ }^{2)}$, Kensuke Sakai ${ }^{1)}$, Yoshifumi Kasuga ${ }^{1)}$, \\ Marie Fukutake $^{1)}$, Yoko Izumi ${ }^{1)}$, Tadashi Matsumoto ${ }^{1)}$, Kazuhiro Minegishi ${ }^{1)}$ and Yasunori Yoshimura ${ }^{1)}$ \\ 1) Department of Obstetrics and Gynecology, Keio University School of Medicine, Tokyo 160-8582, Japan \\ 2) Department of Internal Medicine, Keio University School of Medicine, Tokyo 160-8582, Japan
}

\begin{abstract}
There is a paucity of information on perinatal data regarding gestational diabetes mellitus (GDM) by the new criteria from a real experience because the number of health care associations implementing the new criteria is still limited. The aim of this study is to investigate perinatal features of the new criteria-defined GDM. We reviewed a total of 995 women with singleton pregnancy that underwent GDM screening followed by a diagnostic oral glucose tolerance test (OGTT). All women found to have GDM underwent self-monitoring of blood glucose measurements as well as dietary management. Insulin treatment was initiated when dietary treatment did not achieve the glycemic goal. Of the 995 women, 141 had GDM (14.2\%): 104 with one, 27 with two, and 10 with three abnormal OGTT values. Women with two or three abnormal OGTT values $(2 / 3-\mathrm{AV})$ needed insulin treatment more frequently than those with one abnormal OGTT value (1-AV) $(70.3 \%$ vs $23.1 \%, P<0.0001)$. After adjustment for age, pregravid overweight, gestational weeks at diagnosis, a first-degree family history of diabetes was correlated with the implementation of insulin treatment in women with 1-AV (adjusted odds ratio 3.9; 95\% Confidence Interval 1.7-9.2; $P=0.001$ ). When compared perinatal outcomes between women with normal glucose tolerance and GDM, fetal growth and the occurrence of pregnancy-induced hypertension were comparable between the two groups. Our data suggest that the IADPSG-defined GDM with 1-AV show less severe glucose intolerance, but might be at risk of insulin requirement when a first-degree family history of diabetes exists.
\end{abstract}

Key words: The new consensus criteria, Gestational diabetes mellitus, Large-for gestational age, Gestational hypertension

IN 2010, International Association of Diabetes and Pregnancy Study Group (IADPSG) proposed new criteria for diagnosing and classifying gestational diabetes mellitus (GDM), based on data of the observational hyperglycemia and adverse pregnancy outcomes (HAPO) study [1]. Since the new diagnostic criteria would increase the frequency of GDM diagnosis without a detailed cost-effectiveness analysis, the number of health care associations implementing the new criteria is still limited [2]. Thus, there is a paucity of information on the IADPSG-defined GDM from a real experi-

Submitted Nov. 20, 2013; Accepted Dec. 31, 2013 as EJ13-0496 Released online in J-STAGE as advance publication Jan. 16, 2014 Correspondence to: Kei Miyakoshi, M.D., Department of Obstetrics and Gynecology, Keio University School of Medicine, 35 Shinanomachi, Shinjuku-ku, 160-8582, Tokyo, Japan.

E-mail: kei@z7.keio.jp ence. Especially, clinical significance of GDM by one abnormal value under the IADPSG criteria remains unknown [3].

In Japan, the IADPSG recommendation was adopted in July 2010 [4], and is commonly used in the obstetric practice, although the screening strategy varies in hospitals. With this background, we have investigated perinatal outcomes of the IADPSG-defined GDM in our hospital. First, maternal clinical and metabolic features were compared between women with a single and two or three abnormal oral glucose tolerance test (1- and 2/3-AV) values. Second, factors associated with insulin treatment in women with 1-AV were investigated. Third, perinatal outcomes were compared between women with normal glucose tolerance and IADPSG-defined GDM. 


\section{Materials and Methods}

\section{Subjects}

We conducted a retrospective cohort study of 995 consecutive pregnant Japanese women who were cared for at the perinatal unit of Keio University Hospital from 2011 to 2012. Gestational age was confirmed in the first trimester by crown-rump length measurements. Excluded from this study were women with multi-fetal pregnancies and women whose neonates exhibited congenital anomalies. Women with a medical history indicating either impaired glucose tolerance or diabetes mellitus (DM), or the use of medications known to affect glucose metabolism were also excluded. The research was performed in accordance with the Declaration of Helsinki and informed consent was obtained from patients where appropriate. This study was approved by the institutional review board at Keio University School of Medicine.

\section{GDM screening and glycemic control}

Each woman underwent a two-step screening for GDM: universal early testing and a standard 1-h 50g oral glucose challenge test (GCT) in early and late pregnancy, respectively, based on the clinical recommendation by Japan Society of Obstetrics and Gynecology (JSOG) [4]. The universal early testing included the clinical risk factors, as follows: (1) pregravid overweight (BMI $\geq 25$ ), (2) prior GDM, (3) past history of macrosomia (birth weight $\geq 4000 \mathrm{~g}$ ), (4) a family history of DM in the first-degree relatives, (5) random plasma glucose $\geq 95 \mathrm{mg} / \mathrm{dL}$. Levels of HbA1c and glycoalbumin (GA) were also examined as early testing in our institution (cut-off values: HbAlc $\geq 5.9 \%$, GA $\geq 15.8 \%$ ). Women with positive early testing underwent a diagnostic 75-g OGTT with the measurement of plasma glucose $(\mathrm{mg} / \mathrm{dL})$ and insulin concentration $(\mathrm{mU} / \mathrm{L})$ in the fasting state and at $30 \mathrm{~min}, 1 \mathrm{~h}$, and $2 \mathrm{~h}$ after the glucose load. Plasma glucose and insulin levels were measured by a glucose oxidase method and enzyme immunoassay, respectively. Women with negative early testing or normal OGTT results underwent a standard GCT between 24 and 27 weeks of gestation. If the GCT result exceeded $140 \mathrm{mg} / \mathrm{dL}$, the diagnostic 75-g OGTT was then performed. Based on the IADPSG criteria, GDM was diagnosed if one or more values reached or exceeded the following thresholds: fasting, $92 \mathrm{mg} / \mathrm{dL} ; 1 \mathrm{~h}, 180 \mathrm{mg} / \mathrm{dL} ; 2 \mathrm{~h}, 153 \mathrm{mg} / \mathrm{dL}$ [1]. Overt diabetes in pregnancy was defined as $\mathrm{HbAlc} \geq$
$6.5 \%$ or fasting plasma glucose $\geq 126 \mathrm{mg} / \mathrm{dL}$ or random plasma glucose exceeded $200 \mathrm{mg} / \mathrm{dL}$, the latter needing to be confirmed by one of the former [1]. The normal glucose tolerance (NGT) group comprised women with normal GCT or normal OGTT results.

All women found to have IADPSG-defined GDM underwent self-monitoring of blood glucose (SMBG) measurements as well as dietary management (daily calorie intake: early, $30 \mathrm{kcal} / \mathrm{kg}+150 \mathrm{kcal}$; late, $30 \mathrm{kcal} / \mathrm{kg}$ $+350 \mathrm{kcal}$; if obese, $30 \mathrm{kcal} / \mathrm{kg}$ throughout pregnancy). Dietary management includes three meals and three snacks. Daily capillary glucose profiles were obtained seven times a day under dietary management: fasting, 2 h-post-breakfast, before lunch, 2 h-post-lunch, before dinner, $2 \mathrm{~h}$-post-dinner, and bedtime. Capillary glucose levels were measured with a Medisafe Fit Pro blood glucose meter (Terumo Corporation, Tokyo, Japan). Insulin treatment was initiated when dietary treatment did not consistently maintain fasting and pre-meal capillary glucose $\leq 100 \mathrm{mg} / \mathrm{dL}$ and $2 \mathrm{~h}$ postprandial capillary glucose $\leq 120 \mathrm{mg} / \mathrm{dL}$, respectively. Regular, or rapid acting, and NPH insulin were used to achieve the glycemic target and insulin dose was adjusted according to insulin algorithm based on SMBG values.

\section{Assessment of insulin sensitivity, insulin secretion and beta cell function}

Insulin sensitivity and insulin secretion were evaluated using measurements from the diagnostic OGTT $[5,6]$. The insulin sensitivity was estimated by the whole-body insulin sensitivity index derived from the OGTT (IS OGTT $_{\text {). The IS }}$ OGTT was calculated by the following formula: 10,000/ square root $\left\{\mathrm{Glu}_{0} \times \mathrm{Ins}_{0}\right.$ $\mathrm{x}\left(\mathrm{Glu}_{0}+\mathrm{Glu}_{60}\right.$ × $\left.2+\mathrm{Glu}_{120}\right) / 2$ x $\left(\mathrm{Ins}_{0}+\mathrm{Ins}_{60}\right.$ × 2 $\left.\left.+\operatorname{Ins}_{120}\right) / 2\right\}$, where Glu $\mathrm{y}_{\mathrm{y}}$ and $\mathrm{Ins}_{\mathrm{y}}$ represent plasma glucose $(\mathrm{mg} / \mathrm{dL})$ and insulin values $(\mathrm{mU} / \mathrm{L})$, respectively, at time y min during the OGTT. Insulin secretion was assessed by the ratio of the total area under the insulin curve to the total area under the glucose curve $\left(\mathrm{AUC}_{\mathrm{ins} / \mathrm{glu}}\right)$ during the OGTT. To evaluate beta cell function, we calculated the OGTT-derived disposition index using the following measures: Insulin SecretionSensitivity Index-2 (ISSI-2: the AUC ${ }_{\text {ins/glu }}$ multiplied by $\left.\mathrm{IS}_{\mathrm{OGTT}}\right)$ [7].

\section{Perinatal outcomes}

Maternal characteristics and perinatal outcomes were collected from the patients' hospital records. Gestational hypertension was defined as a blood pres- 
sure of at least 140/90 $\mathrm{mmHg}$ occurring for the first time after mid-pregnancy, without proteinuria or preexisting hypertension. Proteinuria was defined as urinary excretion of at least $0.3 \mathrm{~g}$ in a 24 -hour period. A diagnosis of preeclampsia was made in women who developed gestational hypertension and proteinuria. Using the Japanese standard sex- and parity-specific birthweight percentile curves, birthweight $\geq 90$ th percentile was defined as large-for-gestational age (LGA), and birthweight $<10$ th percentile was designated smallfor-gestational age (SGA). Macrosomia was defined as birthweight above $4000 \mathrm{~g}$.

\section{Statistical analysis}

Data were presented as mean \pm SD or percentage in text and tables, where appropriate. Continuous data were compared between groups by Student's $t$ test. Categorical variables were analyzed with the chisquare test or Fisher's exact test. Statistical analysis was performed using the JMP (SAS Institute, Cary, NC, USA). A value of $P<0.05$ was considered statistically significant.

\section{Results}

Among 995 pregnant women, 141 (14.2\%) were diagnosed to have GDM: 104 with a single abnormal value, 27 with two abnormal values, and 10 with three abnormal values. Of women with GDM, 68 were diagnosed by universal early testing in the first trimester. Two women (2/995 cases, $0.2 \%)$ had overt diabetes in pregnancy.

When compared the clinical features between women with 1 - and 2/3-AV, there were no significant differences in maternal age, pregravid overweight $(\mathrm{BMI} \geq 25)$, prior GDM, a family history of DM, and gestational weeks at diagnosis (Table 1). Compared with 1-AV, women with 2/3-AV showed significantly lower levels of $\operatorname{IS}_{\text {OGTT }}(P<$ 0.01 ), although insulin secretion assessed by $\mathrm{AUC}_{\text {ins/glu }}$ was comparable between the two groups. As a result, levels of ISSI-2 in women with $2 / 3$-AV were significantly lower than those with $1-\mathrm{AV}(P<0.0001)$. To achieve glycemic goal, women with $2 / 3$-AV needed insulin treatment more frequently than those with $1-\mathrm{AV}$ (70.3\% vs $23.1 \%, P<0.0001$, Table 1$)$.

With respect to clinical features in the 1-AV group, there were no significant differences in metabolic phenotypes (insulin sensitivity, insulin secretion, and beta cell function) as well as maternal age, pregravid overweight (BMI $\geq 25$ ), prior GDM, gestational weeks at diagnosis between women with and without insulin treatment (Table 2). Of interest, a family history of DM was more prevalent in women requiring insulin treatment $(41.7 \%$ vs. $15.0 \%, P<0.01$, Table 2$)$. The time point showing abnormal values as well as levels of plasma glucose and insulin at the diagnostic OGTT were not associated with the implementation of insulin treatment (Table 3). After adjustment for age, pregravid overweight, gestational weeks at diagnosis, a family history of DM was significantly correlated with insulin treatment in women with 1-AV (adjusted odds ratio 3.9; 95\% Confidence Interval 1.7-9.2; $P=0.001$ ).

Table 1 Maternal clinical features in women with a single and two/three abnormal oral glucose tolerance test results

\begin{tabular}{|c|c|c|c|}
\hline & & $\begin{array}{l}\text { A single abnormal OGTT result } \\
(\mathrm{n}=104)\end{array}$ & $\begin{array}{l}\text { Two/three abnormal OGTT results } \\
(\mathrm{n}=37)\end{array}$ \\
\hline Maternal age & , years & $35.7 \pm 4.4$ & $35.8 \pm 4.8$ \\
\hline Pregravid BMI & \multirow[t]{4}{*}{, $\mathrm{kg} / \mathrm{m}^{2}$} & $20.7 \pm 3.4$ & $22.3 \pm 5.1^{*}$ \\
\hline Pregravid overweight & & $10(9.6 \%)$ & $8(21.6 \%)$ \\
\hline Prior GDM & & $1(1.0 \%)$ & $2(5.4 \%)$ \\
\hline A family history of diabetes & & $22(21.2 \%)$ & $11(29.7 \%)$ \\
\hline Gestational age at diagnosis & \multirow[t]{2}{*}{, weeks } & $21 \pm 8$ & $22 \pm 7$ \\
\hline Diagnosed before 20 weeks' & & $51(49.0 \%)$ & $17(46.0 \%)$ \\
\hline $\mathrm{HbAlc}$ at diagnosis & \multirow[t]{5}{*}{$\%$} & $5.4 \pm 0.3$ & $5.4 \pm 0.3$ \\
\hline IS $_{\text {OGTT }}$ & & $6.4 \pm 3.0$ & $4.8 \pm 2.7^{\#}$ \\
\hline $\mathrm{AUC}_{\mathrm{ins} / \mathrm{glu}}$ & & $0.34 \pm 0.21$ & $0.41 \pm 0.22$ \\
\hline ISSI-2 & & $2.1 \pm 0.62$ & $1.6 \pm 0.49^{\# \#}$ \\
\hline Insulin treatment & & $24(23.1 \%)$ & $26(70.3 \%)^{\# \#}$ \\
\hline
\end{tabular}

OGTT, oral glucose tolerance test; BMI, body mass index; overweight, BMI $\geq 25$; GDM, gestational diabetes mellitus; $\mathrm{IS}_{\mathrm{OGTT}}$, insulin sensitivity index derived from the oral glucose tolerance test; $\mathrm{AUC}_{\text {ins/glu }}$, the ratio of the total area under the insulin curve to the total area under the glucose curve during the oral glucose tolerance test; ISSI-2, Insulin Secretion-Sensitivity Index-2 (i.e. the $\mathrm{AUC}_{\text {ins/glu }}$ multiplied by $\left.\mathrm{IS}_{\mathrm{OGTT}}\right){ }^{*}, P<0.05{ }^{*}{ }^{\#}, P<0.01{ }^{\# \#}, P<0.0001$ 
Table 2 Maternal characteristics in women with a single abnormal oral glucose tolerance test result

\begin{tabular}{lccc}
\hline & & $\begin{array}{c}\text { Insulin treatment } \\
(\mathrm{n}=24)\end{array}$ & $\begin{array}{c}\text { Dietary management only } \\
(\mathrm{n}=80)\end{array}$ \\
\hline Maternal age &, years & $36.1 \pm 5.0$ & $35.9 \pm 4.3$ \\
Pregravid BMI &, $\mathrm{kg} / \mathrm{m}^{2}$ & $21.1 \pm 2.6$ & $20.5 \pm 3.6$ \\
Pregravid overweight & & $3(12.5 \%)$ & $7(8.8 \%)$ \\
Prior GDM & & $0(0.0 \%)$ & $1(1.25 \%)$ \\
A family history of diabetes & $10(41.7 \%)$ & $12(15.0 \%)$ \\
Gestational age at diagnosis &, weeks & $20 \pm 8$ & $22 \pm 8$ \\
Diagnosed before 20 weeks' pregnancy & & $13(54.2 \%)$ & $38(47.5 \%)$ \\
HbA1c at diagnosis &,$\%$ & $5.4 \pm 0.3$ & $5.4 \pm 0.3$ \\
IS & & $6.6 \pm 3.4$ & $6.3 \pm 2.9$ \\
AUCT & & $0.37 \pm 0.18$ & $0.40 \pm 0.22$ \\
ISSI-2 & & $2.1 \pm 0.74$ & $2.1 \pm 0.58$ \\
\hline OGT & & &
\end{tabular}

OGTT, oral glucose tolerance test; BMI, body mass index; overweight, BMI $\geq 25$; GDM, gestational diabetes mellitus; IS $_{\text {OGTT }}$, insulin sensitivity index derived from the oral glucose tolerance test; $\mathrm{AUC}_{\mathrm{ins} / \mathrm{glu}}$, the ratio of the total area under the insulin curve to the total area under the glucose curve during the oral glucose tolerance test; ISSI-2, Insulin Secretion-Sensitivity Index-2 (i.e. the $\mathrm{AUC}_{\text {ins/glu }}$ multiplied by $\mathrm{IS}_{\mathrm{OGTT}}$ ); ${ }^{\#}, P<0.01$

Table 3 Profiles of the diagnostic oral glucose tolerance test in women with a single abnormal result

\begin{tabular}{|c|c|c|}
\hline & $\begin{array}{c}\text { Insulin treatment } \\
(\mathrm{n}=24)\end{array}$ & $\begin{array}{l}\text { Dietary management only } \\
(\mathrm{n}=80)\end{array}$ \\
\hline \multicolumn{3}{|l|}{ Plasma glucose $(\mathrm{mg} / \mathrm{dL})$} \\
\hline $0 \mathrm{~min}$ & $89.3 \pm 7.1$ & $88.9 \pm 7.4$ \\
\hline $30 \mathrm{~min}$ & $145.2 \pm 17.8$ & $141.7 \pm 22.4$ \\
\hline $60 \mathrm{~min}$ & $152.8 \pm 29.0$ & $153.7 \pm 27.3$ \\
\hline $120 \mathrm{~min}$ & $146.6 \pm 29.6$ & $135.8 \pm 26.2$ \\
\hline \multicolumn{3}{|l|}{ Plasma insulin (mU/L) } \\
\hline $0 \mathrm{~min}$ & $6.4 \pm 3.2$ & $6.6 \pm 4.3$ \\
\hline $30 \mathrm{~min}$ & $58.9 \pm 30.6$ & $60.5 \pm 38.9$ \\
\hline $60 \mathrm{~min}$ & $62.0 \pm 34.7$ & $71.4 \pm 47.3$ \\
\hline $120 \mathrm{~min}$ & $69.2 \pm 37.9$ & $64.3 \pm 41.5$ \\
\hline \multicolumn{3}{|l|}{ Abnormal value of plasma glucose $\mathrm{e}^{\#}$} \\
\hline $0 \mathrm{~min}$ & $9(37.5 \%)$ & $40(50 \%)$ \\
\hline $60 \mathrm{~min}$ & $3(12.5 \%)$ & $14(17.5 \%)$ \\
\hline $120 \mathrm{~min}$ & $12(50 \%)$ & $26(32.5 \%)$ \\
\hline
\end{tabular}

\#, Cases showing abnormal plasma glucose values at each time point in the diagnostic oral glucose tolerance test

The overall perinatal features of this study cohort were as follows: the mean maternal age was $34.9 \pm$ 4.8 years, pregravid BMI $19.8 \pm 4.6$, gestational age at delivery $38.0 \pm 2.4$ weeks, mean birthweight $2841 \pm$ $551 \mathrm{~g}$, and $680(68.0 \%)$ were primiparas. Twenty-five women $(2.5 \%)$ developed gestational hypertension, and $19(1.9 \%)$ preeclampsia. The occurrence of SGA and LGA was 6.9\% (69/995 cases) and 6.0\% (60/995 cases), respectively.

The baseline characteristics and pregnancy outcomes of women with NGT or GDM are shown in Table 4. There were significant differences in maternal age, pregravid overweight, and a family history of DM among subjects with NGT and GDM. With regard to fetal growth, the prevalence of LGA and SGA were comparable between the NGT and GDM groups. Additionally, no significant differences in the occurrence of pregnancy-induced hypertension (i.e. gestational hypertension and preeclampsia) were found between the two groups.

\section{Discussion}

Currently, prospective data on the potential frequency of GDM with the IADPSG criteria has been slow. To the best of our knowledge, this is the first report on perinatal features of women with GDM in the clinical situations that adopted the IADPSG consen- 
Table 4 Baseline characteristics and perinatal outcomes in women with normal glucose tolerance or gestational diabetes mellitus

\begin{tabular}{lccc}
\hline & & NGT $(\mathrm{n}=852)$ & GDM $(\mathrm{n}=141)$ \\
\hline Maternal age & , years & $34.7 \pm 4.8$ & $36.1 \pm 4.6^{*}$ \\
Nulliparous & & $591(69.4 \%)$ & $89(63.1 \%)$ \\
Pregravid BMI &, $\mathrm{kg} / \mathrm{m}^{2}$ & $19.6 \pm 4.7$ & $21.1 \pm 4.00^{\#}$ \\
Pregravid overweight & & $36(4.2 \%)$ & $18(12.8 \%)$ \\
Prior GDM & & $5(0.6 \%)$ & $3(2.1 \%)$ \\
A family history of diabetes & & $57(6.7 \%)$ & $33(23.4 \%)$ \\
\hline Gestational age at delivery &, weeks & $38.1 \pm 2.4$ & $37.8 \pm 2.8$ \\
Birth weight &, $\mathrm{g}$ & $2845 \pm 548$ & $2821 \pm 573$ \\
LGA & & $59(6.9 \%)$ & $10(7.1 \%)$ \\
Macrosomia & $9(1.1 \%)$ & $1(0.7 \%)$ \\
SGA & $53(6.2 \%)$ & $7(5.0 \%)$ \\
Gestational hypertension & & $21(2.5 \%)$ & $4(2.8 \%)$ \\
Preeclampsia & $17(2.0 \%)$ & $2(1.4 \%)$ \\
\hline
\end{tabular}

NGT, normal glucose tolerance, defined as negative screen for gestational diabetes mellitus or normal oral glucose tolerance results. GDM, gestational diabetes mellitus; BMI, body mass index, overweight, $\mathrm{BMI} \geq 25$; LGA, large-for-gestational age, defined as birth weight $\geq 90$ th percentile for gestational age; SGA, small-for-gestational age, defined as birth weight $<10$ th percentile for gestational age; Macrosomia, birth weight $\geq 4000 \mathrm{~g}$. *, $P<0.05$;,$P<0.001$; ${ }^{\# \#}, P<0.0001$.

sus criteria. In our institution, the prevalence of GDM by the former JSOG criteria was $2.3 \%$, as previously reported [8]. Compared with the former situations, the IADPSG criteria lead to an increase in the frequency of GDM diagnosis in our hospital. Additionally, the majority (104/141 cases, 74\%) of women with GDM showed 1-AV, which was consistent with data in the complete HAPO cohort [1]. Our results demonstrated that the frequency of GDM would increase significantly with the IADPSG criteria, mainly by those with 1-AV.

The implementation of insulin treatment was based on daily glucose profile during dietary management in our hospital. In this retrospective analysis, more women with $2 / 3-\mathrm{AV}$ needed the addition of insulin treatment to achieve the glycemic control, compared with 1-AV. With respect to beta cell function, levels of ISSI-2 in women with 2/3-AV were significantly lower than those with 1-AV. The level of beta cell function is associated with the severity of glucose intolerance in GDM [9]. Additionally, our previous investigation demonstrated that beta cell dysfuntion in women with 2/3-AV appeared more severe than those with 1-AV [10]. Taken altogether, our data indicated women with 2/3-AV had more severe levels of glucose intolerance, compared with 1-AV.

Approximately one-quarter of women with 1-AV needed the insulin treatment. Most women with the IADPSG-defined GDM have 1-AV and factors asso- ciated with insulin treatment are needed in the clinical practice. The insulin treatment did not depend on which glucose result met or exceeded single IADPSGdefined OGTT threshold. When analyzed maternal characteristics, a family history of DM was correlated with the induction of insulin treatment. Of women with the IADPSG-defined GDM, therefore, those with 1-AV appear less severe glucose intolerant, but might be at risk of insulin requirement when a family history of DM exists.

There were significant differences in pregravid overweight, prior GDM, and a family history of DM among subjects with NGT and GDM, as were noted in the former situations [11]. Of note, no significant differences were found in the occurrence of perinatal outcome including LGA and pregnancy-induced hypertension between the NGT and GDM groups in this study cohort. Previous analysis based on reevaluated data before the adoption of the IADPSG criteria has shown increased risk of the development of LGA and gestational hypertension in the IADPSG-defined GDM [12]. Additionally, Black et al. have shown that IADPSGdefined GDM could be at risk of adverse outcomes including LGA, gestational hypertension and shoulder dystocia/birth injury [13]. To date, two trials have demonstrated the advantages of treatment for women with mild degree glucose intolerance, although inclusion criteria for the trial were different from the IADPSG rec- 
ommendation [14-16]. Our results suggest that intervention for women with IADPSG-defined GDM could contribute to the improvement of pregnancy outcomes, although further studies on the clinical and cost-effective management are needed.

Benefit of treatment of IADPSG-defined GDM remains unknown because no randomized control trial has been conducted using the IADPSG criteria and additional well-designed trial and other clinical studies will be needed to determine the optimal treatment targets [1]. In the clinical settings, therefore, appropriate management of IADPSG-defined GDM remains unclear. Our data is only a local experience but could be useful for other healthcare professionals considering the IADPSG criteria.

\section{Acknowledgment}

We thank Ms. Melinda Murphy for her helpful suggestions in preparing manuscript.

\section{Disclosure}

Authors declare no conflict of interest with regard to this manuscript.

\section{References}

1. Metzger BE, Gabbe SG, Persson B, Buchanan TA, Catalano PA, et al. (2010) International association of diabetes and pregnancy study groups recommendations on the diagnosis and classification of hyperglycemia in pregnancy. Diabetes Care 33: 676-682.

2. Holt RI, Coleman MA, McCance DR (2011) The implications of the new International Association of Diabetes and Pregnancy Study Groups (IADPSG) diagnostic criteria for gestational diabetes. Diabet Med 28: 382-385.

3. Langer O, Umans JG, Miodovnik M (2012) The proposed GDM diagnostic criteria: a difference, to be a difference, must make a difference. J Matern Fetal Neonatal Med 26: 111-115.

4. Minakami H, Hiramatsu Y, Koresawa M, Fujii T, Hamada $\mathrm{H}$, et al. (2011) Guidelines for obstetrical practice in Japan: Japan Society of Obstetrics and Gynecology (JSOG) and Japan Association of Obstetricians and Gynecologists (JAOG) 2011 edition. J Obstet Gynaecol Res 37: 1174-1197.

5. Matsuda M, DeFronzo RA (1999) Insulin sensitivity indices obtained from oral glucose tolerance testing: comparison with the euglycemic insulin clamp. Diabetes Care 22: 1462-1470.

6. DeFronzo RA, Matsuda M (2010) Reduced time points to calculate the composite index. Diabetes Care 33: e93.

7. Retnakaran R, Shen S, Hanley AJ, Vuksan V, Hamilton JK, et al. (2008) Hyperbolic relationship between insulin secretion and sensitivity on oral glucose tolerance test. Obesity 16: 1901-1907.

8. Miyakoshi K, Tanaka M, Ueno K, Uehara K, Ishimoto $\mathrm{H}$, et al. (2003) Cutoff value of $1 \mathrm{~h}, 50 \mathrm{~g}$ glucose challenge test for screening of gestational diabetes mellitus in a Japanese population. Diabetes Res Clin Pract 60:
63-67.

9. Saisho Y, Miyakoshi K, Tanaka M, Shimada A, Ikenoue $\mathrm{S}$, et al. (2010) Beta cell dysfunction and its clinical significance in gestational diabetes. Endocr J 57: 973980.

10. Miyakoshi K, Saisho Y, Tanaka M, Shimada A, Itoh $\mathrm{H}$, et al. (2011) Pancreatic beta cell function in women with gestational diabetes defined by new consensus criteria. Diabetes Care 34: e8.

11. Miyakoshi K, Tanaka M, Matsumoto T, Hattori Y, Ueno $\mathrm{K}$, et al. (2004) Hypertensive disorders in Japanese women with gestational glucose intolerance. Diabetes Res Clin Pract 64: 201-205.

12. Matsumoto T, Miyakoshi K, Minegishi K, Tanaka M, Yoshimura Y (2012) Fetal growth and gestational hypertension in women classified as gestational diabetes mellitus defined by the new consensus criteria only. Acta Obstet Gynecol Scand 91: 272-273.

13. Black MH, Xiang AH, Sacks DA, Lawrence JM (2010) Clinical outcomes of pregnancies complicated by mild gestational diabetes mellitus differ by combinations of abnormal oral glucose tolerance test values. Diabetes Care 33: 2524-2530.

14. Crowther CA, Hiller JE, Moss JR, McPhee AJ, Jeffries WS, et al. (2005) Effect of treatment of gestational diabetes mellitus on pregnancy outcomes. $N$ Engl J Med 352: 2477-2486.

15. Landon MB, Spong CY, Thom E, Carpenter MW, Ramin SM, et al. (2009) A multicenter, randomized trial of treatment for mild gestational diabetes. $N$ Engl J Med 361: 1339-1348.

16. Landon MB (2010) Is there a benefit to the treatment of mild gestational diabetes mellitus? Am J Obstet Gynecol 202: 649-653. 